\title{
Complex management of electrical storm in a patient with non-ischemic dilated cardiomyopathy
}

\author{
Ovidiu Mitu ${ }^{*}, 1,2$, Mircea Balasanian ${ }^{1,2}$, Cristian Stătescu ${ }^{1,2}$, Mihaela Grecu², Anca \\ Dabija $^{2}$, Alexandra lovoaia², Ecaterina Strîmbeanu², Cătălina Arsenescu- \\ Georgescu $^{1,2}$
}

1“Grigore T. Popa” University of Medicine and Pharmacy, lasi, Romania, 2“Prof. Dr. George I. M. Georgescu" Institute of Cardiovascular Diseases, Iasi, Romania

\begin{abstract}
Electrical storm represents a major clinical emergency characterized by electrical heart instability with several recurrent episodes of ventricular arrhythmias over a short period of time. We present the case of a 67year old male, hypertensive, diabetic who was referred to the cardiology department after a resuscitated cardiac arrest through sustained ventricular tachycardia. The echocardiography revealed a severe aspect of dilated cardiomyopathy and fibrotic aspect of inferior-posterior wall of the left ventricle. The ischemic coronary cause has been ruled out by coronary computed tomography. An implantable cardioverter defibrillator (ICD) was implanted. One day after, the electrical storm and hemodynamically stability worsened requiring high dosage of amiodarone, putting therapies of ICD on "off" and the intubation of the patient. Taking all into account, an urgent electrophysiological study was performed and led to complex radiofrequency catheter ablation of the substrate of the lateral, medium-ventricular wall. Afterwards, the electrical storm did not reappear, the ICD therapies were put on "on" and the patient was rapidly extubated, being discharged with pharmacological recommendations, the milestone being the anti-arrhythmic treatment. Though the treatment of electrical storm is complex but still unclearly defined, we emphasize the need of applying all the therapeutical measures and having a prompt response to this life-threatening condition.
\end{abstract}

Keywords: electrical storm, catheter ablation, implantable cardioverter defibrillator, anti-arrhythmic, ventricular tachycardia, non-ischemic dilated cardiomyopathy

\section{Introduction}

The term "electrical storm" represents a state of electrical heart instability characterized by several recurrent episodes of ventricular arrhythmias over a short period of time [1, 2]. Both ventricular tachycardia (VT) and ventricular fibrillation (VF) could be the triggers for this life-threating condition. In the last decade, the use of implantable cardioverter

Received: January 2017; Accepted after review: June 2017; Published: June 2017.

*Corresponding author: Ovidiu Mitu, MD, "Grigore T. Popa" University of Medicine and Pharmacy, lasi, 16 Universitatii St, 700115, lasi, Romania.

E-mail: ovidiu.mitu@umfiasi.ro defibrillators (ICDs) has significantly increased and improved the survival of patients with malignant ventricular arrhythmias. The most widely accepted definition for the electrical storm is represented by at least three different episodes of arrhythmia leading to ICD therapies (antitachycardia pacing or internal shock) over a 24-hour period [1, 3].

Electrical storm affects mainly patients with advanced dilated cardiomyopathy, either ischemic or non-ischemic, as well as patients with different structural heart diseases [4]. This condition represents a clinical emergency that require hospitalization. Besides monitoring the hemodynamic and metabolic parameters, the therapeutic management could be very extensive, from pharmacological medications (anti-arrhythmics, sedation) to ICD 
reprogramming and catheter ablation. Thus, we present the case of a patient with nonischemic dilated cardiomyopathy who benefited of all modern cardiologic treatment in order to control and cure the refractory electrical storm.

\section{Case report}

We present the case of a 67-year old male, overweight (body mass index at 27.5 $\mathrm{kg} / \mathrm{m}^{2}$ ), former smoker, who was admitted to the cardiology clinic for accusing acute onset of rapid, regular short episodes of palpitations. Two months before, he presented a resuscitated cardiac arrest through sustained VT and still under anti-arrhythmic treatment, amiodarone in this case, the patient experienced multiple episodes of VT and syncope.

From the patient medical history, we retained the following diagnoses: arterial hypertension grade 3 , type 2 diabetes mellitus complicated with chronic kidney disease and right aorto-femural bypass. Thus, the patient was under chronic medical treatment with antihypertensive, antiplatelet drugs, statin, oral anti-diabetics and amiodarone that was recently introduced.

At admission, the patient was hemodynamically stable, no edema, but presenting shortness of breath at short distances of walking. The complete blood count revealed mild anemia (hemoglobin 11.7 $\mathrm{g} \%$ ), no inflammatory syndrome or myocardial cytolysis, normal lipid and hepatic profile, hyperglycemia (111 mg\%), decreased renal function (glomerular filtration rate - 28 $\left.\mathrm{ml} / \mathrm{min} / 1.73 \mathrm{~m}^{2}\right)$, mild hyponatremia (130 $\mathrm{mmol} / \mathrm{l})$, normal serum potassium level. The resting electrocardiogram showed sinus rhythm, $70 \mathrm{bpm}$, pattern of left ventricular hypertrophy and one isolated premature ventricular complex which appeared immediately after a QRS complex, having a positive prognosis for VT (Figure 1).

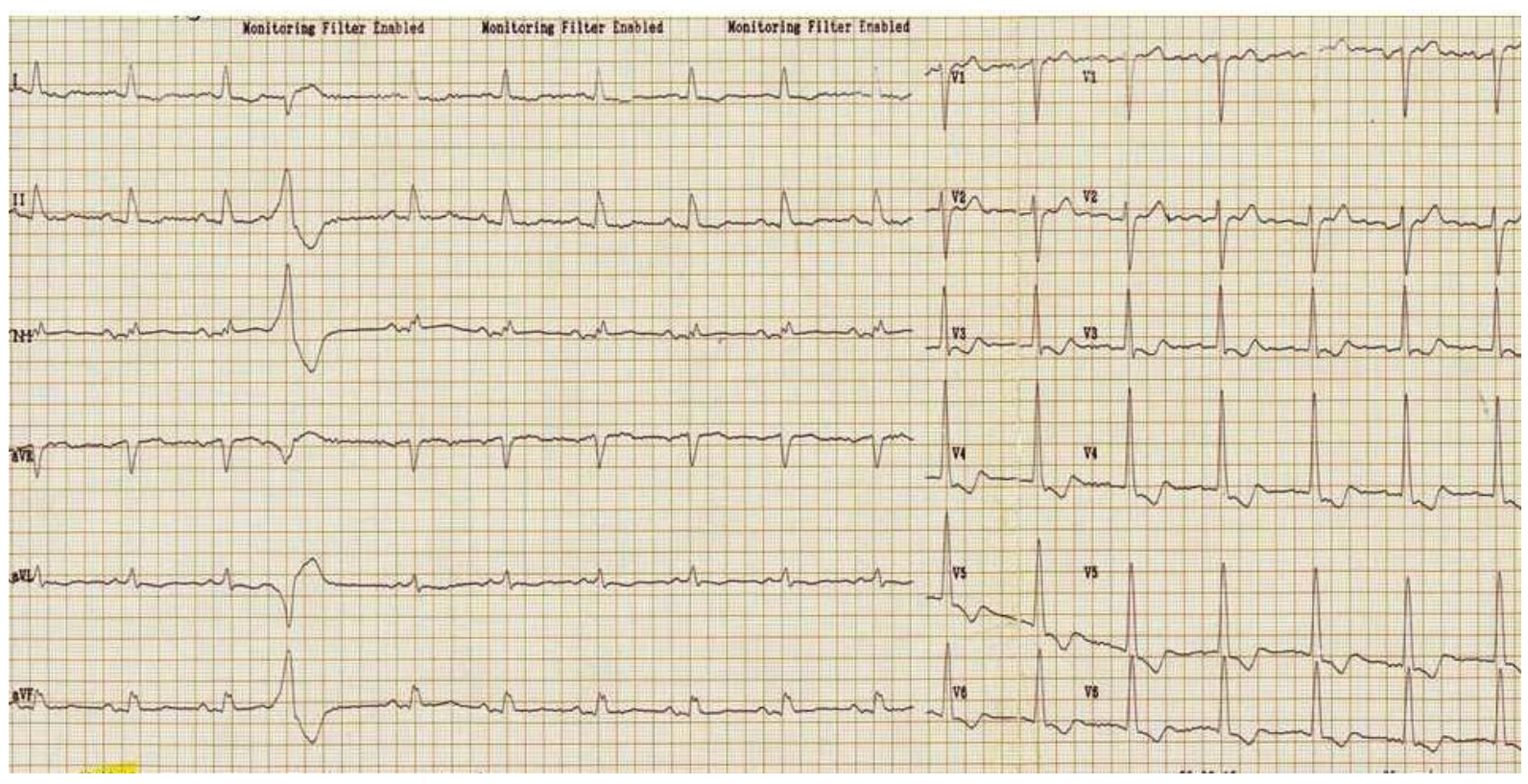

Fig. 1. Resting ECG: sinus rhythm, 70 bpm, pattern of left ventricular hypertrophy and one isolated premature ventricular complex with extreme axial deviation (seen only in unipolar leads)

On transthoracic echocardiography, we found an aspect of dilated cardiomyopathy (end-diastolic left ventricular diameter $99 \mathrm{~mm}$, left ventricular volume $320 \mathrm{ml}$, left ventricular ejection fraction $28 \%$ by Simpson biplane method), biatrial dilatation, mild left ventricular hypertrophy, aortic atheromatosis, no pericardial effusion, no indirect signs of pulmonary arterial hypertension, moderate mitral regurgitation and thin, hyperechoic inferior-posterior wall - fibrotic aspect (Figure 2). 

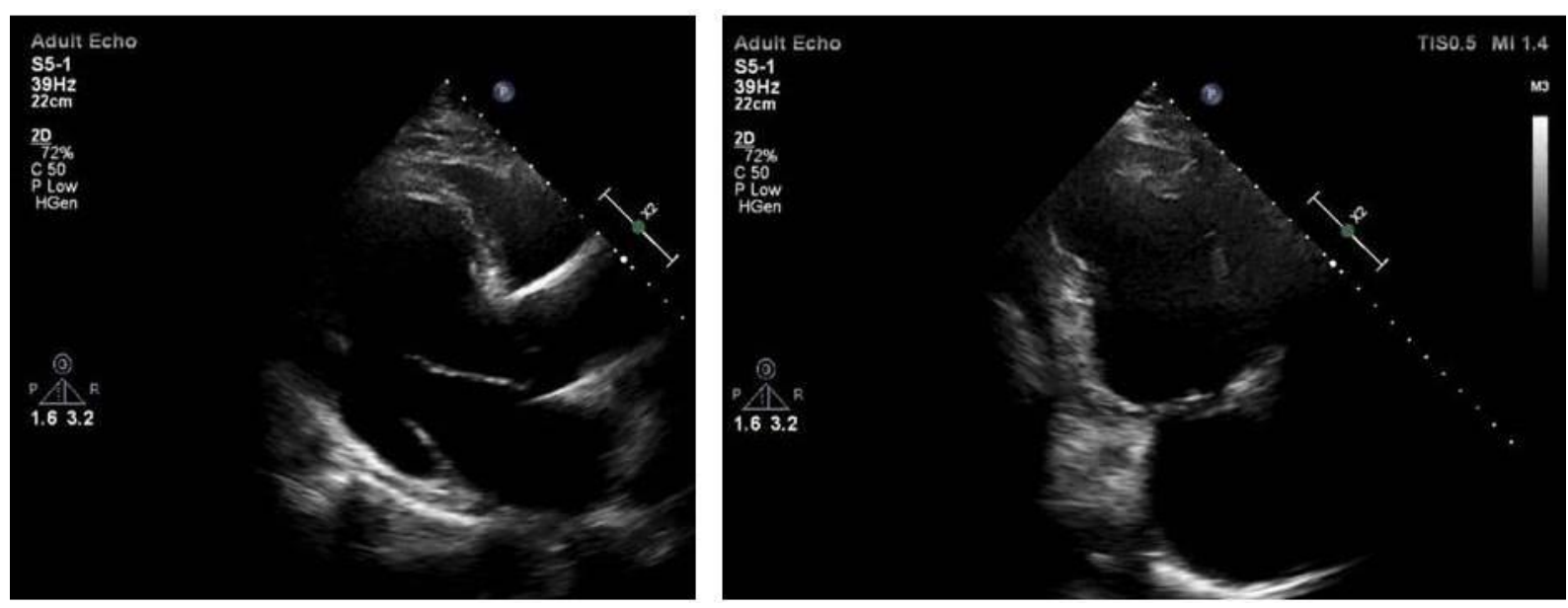

Fig. 2. Transthoracic echocardiography: aspect of dilated cardiomyopathy; thin, hyperechoic inferior-posterior wall - fibrotic aspect

Due to the previous resuscitated cardiac arrest, continuation of malignant tachyarrhythmia and echocardiographic aspect, an ICD with DDD pacing function was implanted (Figure 3). During the positioning of the electrode in the right ventricle, the patient experienced five episodes of sustained VT which required external electrical shocks and intravenous lidocaine.

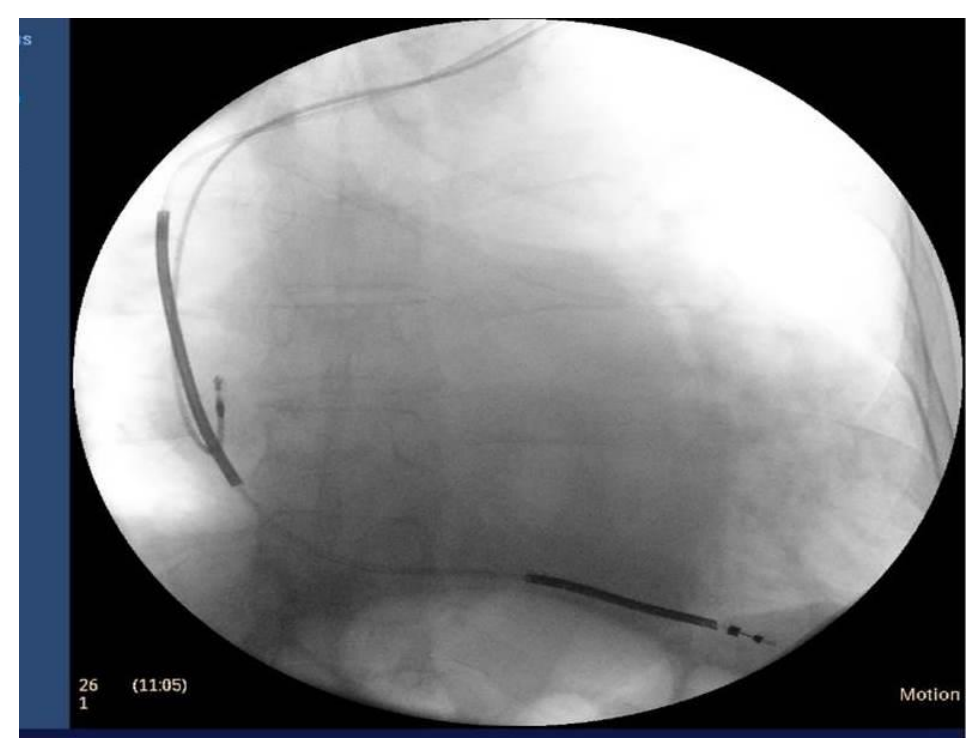

Fig. 3. ICD implantation

However, one day after the ICD procedure, the patient received more than 5 internal electric shocks expression of sustained VT which required putting the therapies of ICD on "off" (Figure 4). Since the patient had multiple cardiovascular risk factors for coronary artery disease plus taking into consideration the echocardiographic aspect and the presence of ventricular arrhythmia, a coronary computed tomography was performed. The exam revealed an increased Agatston coronary calcium score $1177 \mathrm{HU}$ and multiple plaques on all coronary arteries but hemodynamically insignificant (less than 50\%). Nonetheless, the electrical storm continued and required multiple external electric shocks, high amiodarone doses, intravenous lidocaine and magnesium sulfate. The evolution was unfavorable and finally the patient was intubated and sedated due to the continuous hemodynamic instability. 


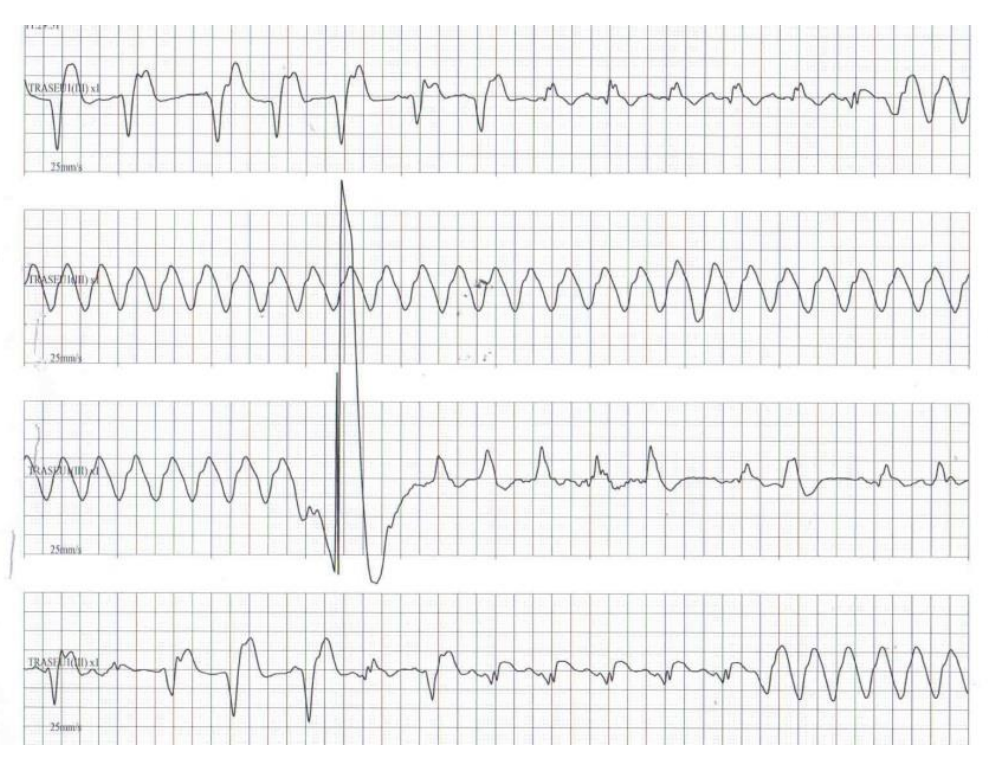

Fig . 4. Monitoring ECG: Internal electrical shock terminating an episode of VT

Since under the ICD and maximal medical treatment the ventricular tachyarrhythmia was not resolved, an urgent electrophysiological study was performed. The ECG before the study showed severe intracardiac conduction with right bundle branch block that was not present at admission. This may be explained by the hemodynamic changes (the patient was intubated and received several shocks) and the multiple antiarrhythmic medications that the patient received (Figure 5). The study revealed severe infra-hisian conduction disease and VT of the free lateral wall of the left ventricle with initial induction of at least three morphologies of VT (Figures $6-8$ ). The regular rhythm and increased frequency, the wide QRS complex, the electric concordance, the R-wave peak time in lead DII $\geq 50 \mathrm{msec}$ or the presence of $R$ wave in aVR lead are characteristics for VT morphology. In the same intervention, a complex radiofrequency catheter ablation of the substrate of the lateral, medium-ventricular wall was performed, leaving a minor residual myocardial substrate due to the extensive intramural myocardial fibrosis and thinness of the posterior ventricular wall.

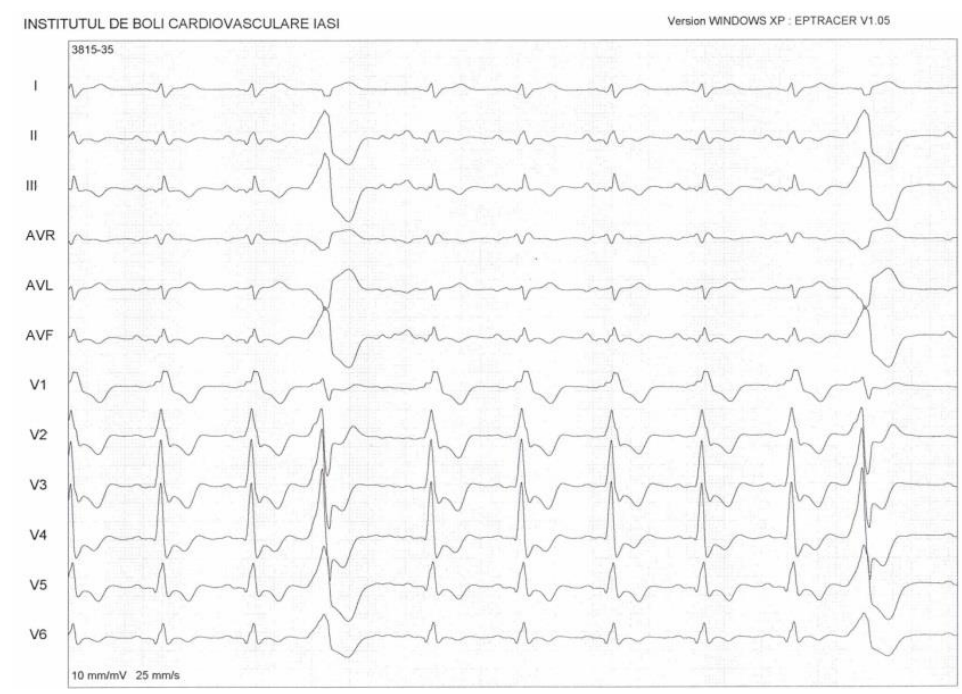

Fig. 5. ECG before electrophysiological study: sinus rhythm, 70 bpm, new right bundle branch block, isolated PVCs with morphology of left bundle branch block with severe intracardiac conduction 


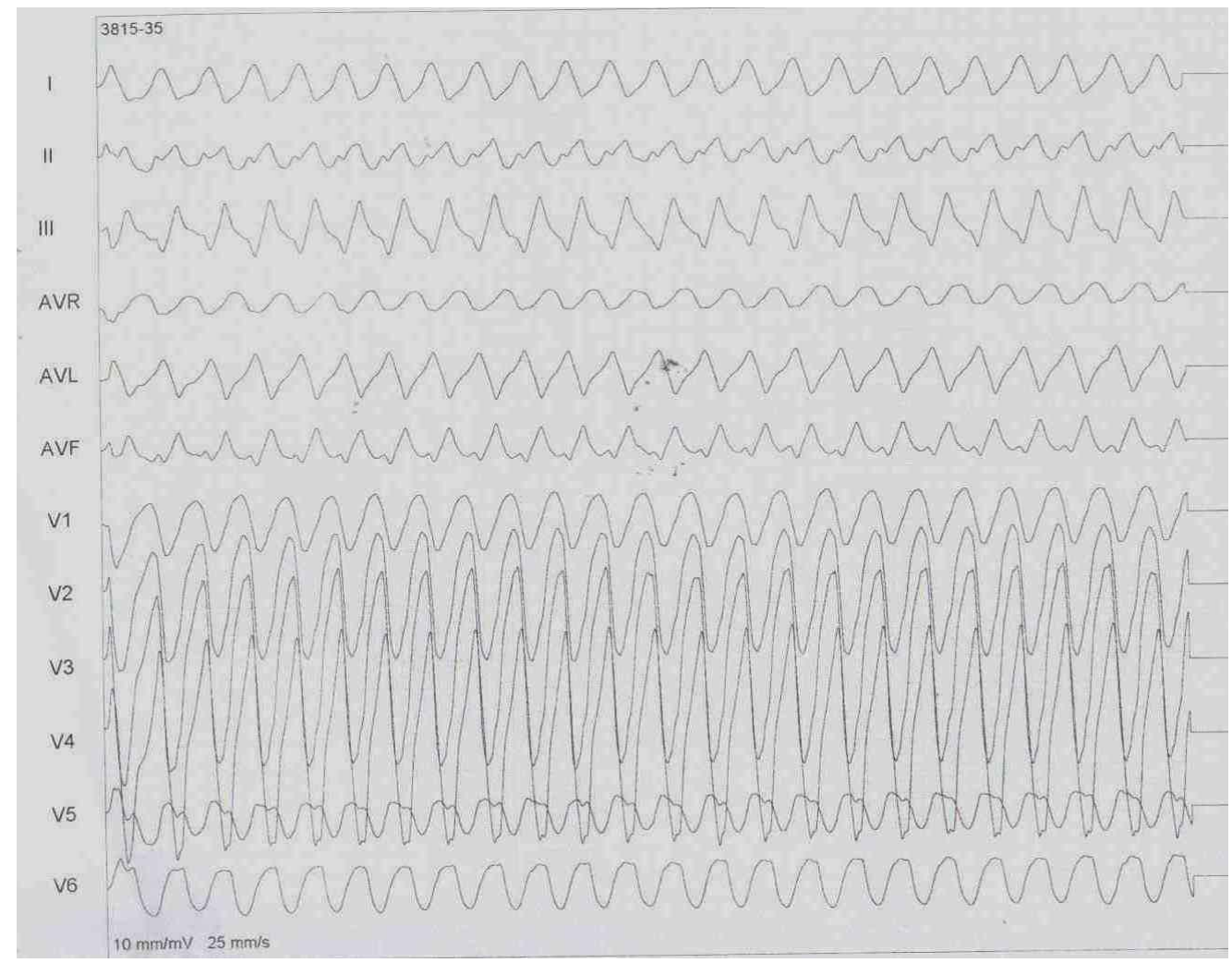

Fig. 6. Electrophysiological study: induction of one type of VT morphology (monomorphic VT with aspect of left bundle branch block, $180 \mathrm{bpm}$, negative concordance, superior axe - possible localization in the posterior interventricular septum)

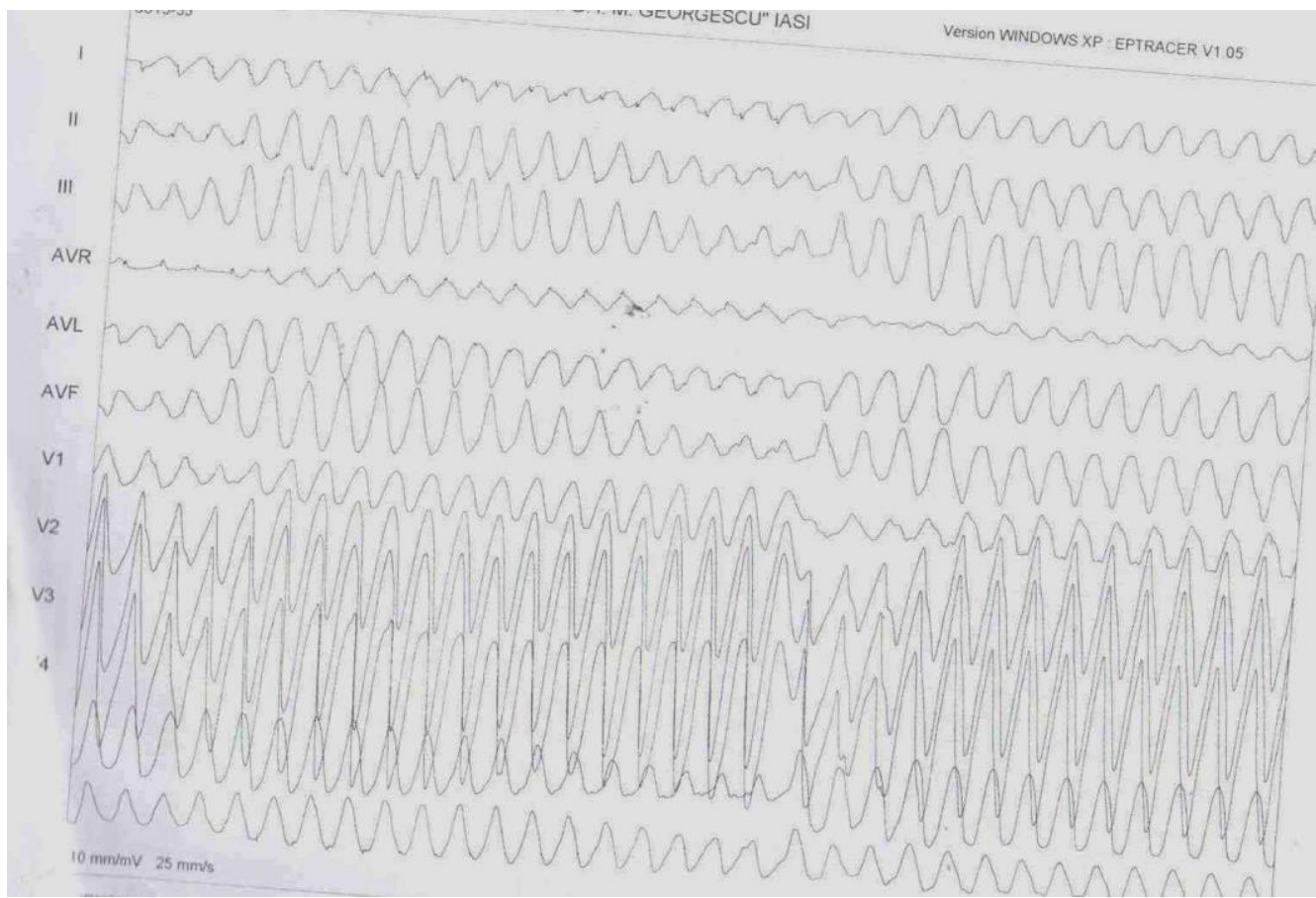

Fig. 7. Electrophysiological study: induction of another type of VT morphology (monomorphic VT with aspect of right bundle branch block, $200 \mathrm{bpm}$, superior axe - possible localization in the postero-lateral wall of the left ventricle) 


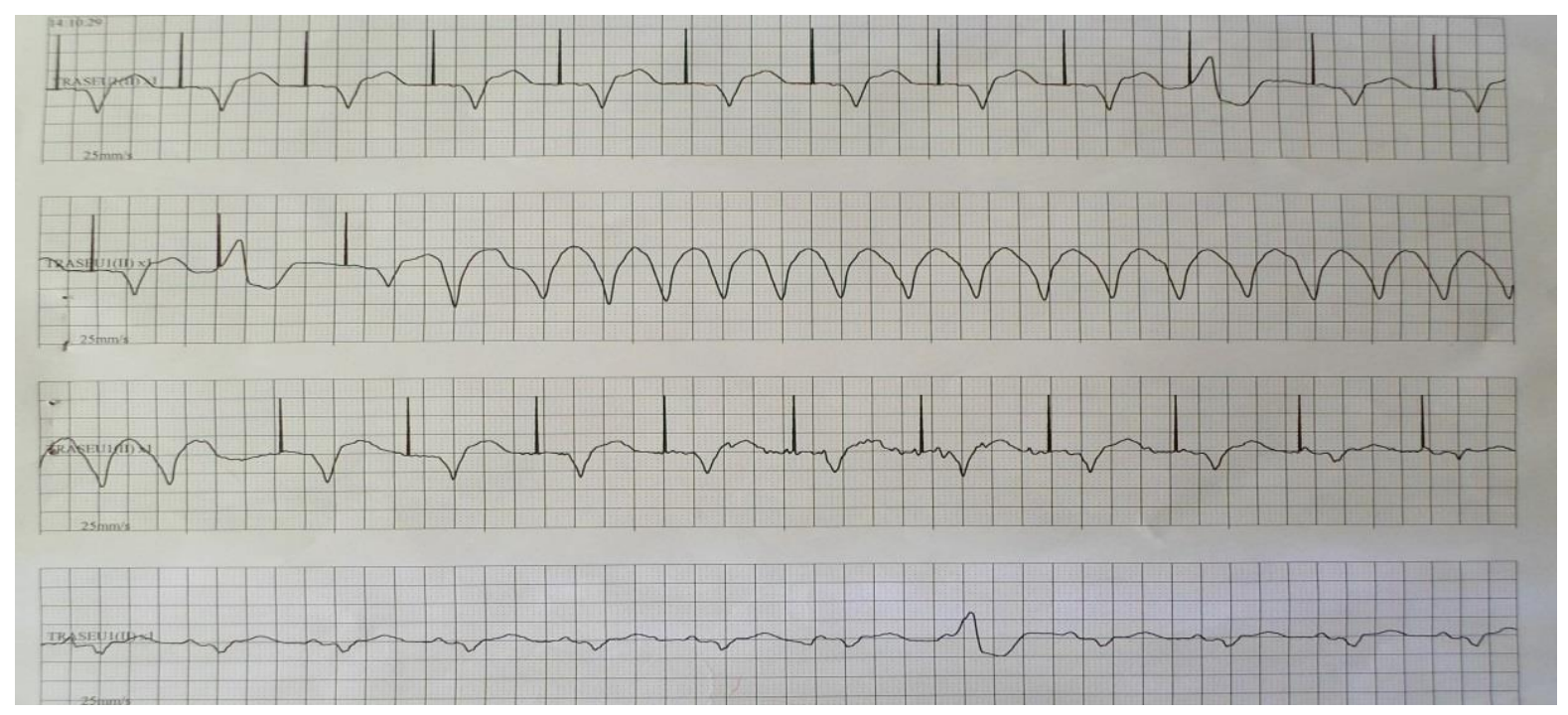

Fig. 8. Electrophysiological study: induction of another type of monomorphic VT morphology, $160 \mathrm{bpm}$

Afterwards, the electrical storm did not reappear (only rare and short episodes of selflimited non-sustained VT), the ICD therapies were put on "on" and the patient became hemodynamically stable being extubated three days after the catheter ablation. A couple of days after, he was discharged from the hospital remaining with chronic medical treatment consisting of: amiodarone (in maintenance dose), diuretics (furosemide and spironolactone), antiplatelet therapy (clopidogrel), beta-blocker, angiotensinconverting enzyme inhibitor, oral iron therapy, omega-3 polyunsaturated fatty acids and gastric protection. Two months after the electrical storm, the evolution of the patient was good without any complications or tachyarrhythmic episodes.

\section{Discussions}

We have presented the case of a patient with electrical storm in whom only the catheter ablation managed to suppress the malignant ventricular arrhythmia despite the maximal pharmacological treatment and ICD reprograming. In addition, this case is more particular since initially he became hemodynamically unstable and was mechanically ventilated but the recovery was favorable after the ablation, the patient being extubated and safely discharged.
With a continuous increase of ICDs, the number of patients with electrical storm is growing since $10 \%$ to $25 \%$ of patients with ICD implanted for secondary prevention of sudden cardiac death develop this malignant condition [1]. However, the incidence is lower, less than $5 \%$, in patients with ICD for primary prevention [5].

The electrical storm is associated with higher rates of mortality in both primary and secondary prevention [6]. However, data is insufficient yet to affirm whether repeated ICD shocks and ventricular tachyarrhythmias contribute to cardiac mortality or are the consequence of an impaired cardiac status [1]. A recent review showed that the substrate of electrical storm is due to a scar-related reentry mechanism in more than $80 \%$ of cases [7]. During an electrical storm, most arrhythmic episodes are due to monomorphic VT (more than $80 \%$ ), VF alone accounts for $1-21 \%$ of episodes, mixed VT-VF is also rare and the incidence of polymorphic VT is about 2-8\% [1]. In our case, the patient presented the latter form of arrhythmia probably due to the dilated cardiomyopathy and the extensive lateral wall fibrosis.

The management of our patient was made according to the European guidelines and standards. Firstly, the ICD was implanted since the patient had dilated cardiomyopathy and recent history of hemodynamically not tolerated VT, plus a good functional condition (class I of recommendation) [8]. Secondly, the 
coronary evaluation has been performed being indicated in patients with dilated cardiomyopathy and new onset of ventricular arrhythmias (class I of recommendation) [8]. Nonetheless, the recommendation refers to stable patients but we considered important to check the coronary status taking into consideration the medical history of the patient and the persistence of arrhythmia. Even though the coronary angiography is the best method for detecting coronary artery disease [9] we have chosen computed coronary tomography due to the renal impairment, lower dose of contrast and for it is more comfortable for the patient than a coronarography. The patient did not present significant coronary lesions at the moment of the current investigation but this cannot exclude a possible old ischemic substrate of the arrhythmia, the echocardiographic aspect being in favor of this explanation. Finally, the current guidelines recommend "urgent" catheter ablation in patients with electrical storm resulting in ICD shocks (class I of recommendation) [8]. The same indication was for the use of amiodarone but our patient did not response entirely to the pharmacological treatment only.

Though ICD terminates effectively VT episodes, it does not prevent arrhythmia recurrence. Catheter ablation has become a useful and suitable strategy for treating persistent ventricular arrhythmias. In most cases, patients with VT in the context of scarrelated tissue and cardiomyopathy present several reentry circuits and different morphologies. In such cases, the cardiac mapping allows the identification and fixation of ablation spots. Moreover, pace-mapping along with the identification of mid-diastolic potentials increase the success rates for VT ablation [10]. However, there is not much data and definitely not on large number of patients to affirm with certainty that catheter ablation represents the best therapeutic solution [11]. However, catheter ablation has significantly decreased the ICD therapies compared to ICD only in secondary prevention [12]. Moreover, patients with prior myocardial infarction who had amiodarone plus catheter ablation had lower event rates than patients treated only with amiodarone [13]. These studies support the therapeutic measures that were chosen in our case and our results bring valuable data for clarifying in the future the best management in such patients.

The cardiac magnetic resonance (CMR) is excellent for evaluating the structure and function of the heart. As well, it represents a reliable method for detecting myocardial scarring in patients with dilated cardiomyopathy [8]. In our case, the CMR would have brought useful information especially before the catheter ablation procedure by showing the areas of myocardial fibrosis and thickness of the ventricular walls. Nonetheless, the acute and fast evolution of the electrical storm forced us to perform the ICD and catheter ablation without having the CMR examination.

Other particularities of the present study consist in the association of comorbidities since the patient had type 2 diabetes mellitus complicated both with micro and macroangiopathy. Renal function was closely monitored since the risk of severe impairment was very high due to contrast computed tomography, catheter ablation procedure or the hemodynamically instability. Fortunately, the serum biochemical parameters did not alter significantly during the hospitalization. As well, it is surprisingly that the patient did not present episodes of tachyarrhythmia earlier in life since the left ventricle was severely dilated and the patient was at first manifestations of acute heart failure. The prognosis of this patient remains reserved taking into consideration all the cardiac and non-cardiac comorbidities but the quality of life will definitely be increased. Moreover, the risk of malignant arrhythmia has been reduced after performing the catheter ablation but has not been totally removed as dilated cardiomyopathy is a negative prognosis factor for such conditions and there remained a minor residual myocardial substrate that may be a trigger for VT. On the other hand, the presence of ICD will assure a more safely management.

\section{Conclusions}

Electrical storm represents a lifethreatening clinical condition which is associated with low survival rates and impaired quality of life. Although the physiopathology of this phenomenon is not completely clarified, 
these patients require aggressive and prompt interventions. Besides optimal pharmacological therapy, recent progress in nonpharmacological treatment, such as ICD monitoring and catheter ablation, has significantly improved the cardiovascular outcomes, especially if they are combined with modern investigations such as echocardiography and CMR. Our case has

\section{References}

1. Sagone A. Electrical Storm: Incidence, Prognosis and Therapy. J Atr Fibrillation 2015; 8(4):1150.

2. Maruyama M. Management of electrical storm: The mechanism matters. J Arrhythm 2014; 30:242-249.

3. Hohnloser SH, Al-Khalidi HR, Pratt CM, et al. Electrical storm in patients with an implantable defibrillator: incidence, features, and preventive therapy: insights from a randomized trial. Eur Heart J 2006; 27(24):3027-3032.

4. Conti S, Pala S, Biagioli V, et al. Electrical storm: A clinical and electrophysiological overview. World J Cardiol 2015; 7(9):555-561.

5. Sesselberg HW, Moss AJ, McNitt S, et al. Ventricular arrhythmia storms in postinfarction patients with implantable defibrillators for primary prevention indications: a MADIT-II substudy. Heart Rhythm 2007; 4(11):13951402.

6. Gao D, Sapp JL. Electrical storm: definitions, clinical importance, and treatment. Curr Opin Cardiol 2013; 28(1):72-79.

7. Nayyar S1, Ganesan AN, Brooks AG, Sullivan T, Roberts-Thomson KC, Sanders P. Venturing into ventricular arrhythmia storm: a systematic review and meta-analysis. Eur Heart $J$ 2013; 34(8):560-571. integrated all these possible therapeutical measures and further research will clarify the specific role and timing of each intervention.

\section{Conflict of interest}

The authors declare that they have no competing interests.

8. Priori SG, Blomström-Lundqvist C, Mazzanti A, et al. 2015 ESC Guidelines for the management of patients with ventricular arrhythmias and the prevention of sudden cardiac death. Eur Heart J 2015; 36(41):27932867.

9. Radu R, Mitu O, Nedelciuc I, ArsenescuGeorgescu C. Severe coronary spasm and poor relationship with other non-invasive methods of detecting coronary spasm. Rom $J$ Cardiol 2014; 24(1):381-382.

10. Ciudin R. Interventional treatment in cardiac arrythmias. In Apetrei E (Ed.) Clinical Cardiology. Bucharest: Callisto Medical Ed; 2015:574-587.

11. Chen M. Ventricular tachycardia ablation and substrate modification in ICD patients with electrical storm. J Biomed Res 2015; 29(1):5255.

12. Reddy VY, Reynolds MR, Neuzil $P$, et al. Prophylactic catheter ablation for the prevention of defibrillator therapy. N Engl J Med 2007; 357(26):2657-2665.

13. Kuck KH, Schaumann A, Eckardt $L$, et al. Catheter ablation of stable ventricular tachycardia before defibrillator implantation in patients with coronary heart disease (VTACH): a multicentre randomised controlled trial. Lancet 2010; 375(9708):31-40. 